\title{
Malformaciones linfáticas del ducto torácico cervical tratadas mediante escleroterapia
}

\section{Lymphatic malformations of the cervical thoracic duct treated by sclerotherapy}

\author{
Catalina S. Kychenthal-Loyola ${ }^{1}$ María J. Irarrázaval-Mainguyague¹, Sebastián A. Larraín-Castellón ${ }^{1}$, \\ Josefina A. Gazmuri-Loyola ${ }^{1}$, Alejandro Zavala-Busquets ${ }^{2}$ y María S. Loyola-Zunino ${ }^{3 *}$ \\ ${ }^{1}$ Escuela de Medicina, Pontificia Universidad Católica de Chile; ${ }^{2}$ Servicio de Cirugía Pediátrica; ${ }^{3}$ Servicio de Radiología Intervencional, Centro de
} Anomalías Vasculares UC, Facultad de Medicina, Pontificia Universidad Católica de Chile. Santiago, Chile

\begin{abstract}
Resumen
Introducción: Las malformaciones vasculares de bajo flujo linfáticas quísticas (ML) son lesiones caracterizadas por tejido linfático desorganizado y la formación de cavidades quísticas. A pesar de ser benignas, pueden complicarse o causar sintomatología, y requerir tratamiento. El manejo inicial de elección es la escleroterapia percutánea con guía ecográfica. Existen casos poco frecuentes de estas lesiones a nivel del cayado del ducto torácico, las cuales se presentan como una masa quística cervical baja. Objetivo: Describir, a propósito de dos casos, la identificación y el manejo de ML ubicadas en la desembocadura del ducto torácico. Material y métodos: Se realizó un análisis retrospectivo de cohorte no concurrente, analizando dos casos de pacientes tratados en nuestro centro con escleroterapia con doxiciclina y polidocanol por ML supraclaviculares izquierdas. Se describen en detalle el diagnóstico y la técnica utilizada en el procedimiento. Resultados: En ambos casos se confirmó el diagnóstico de malformación linfática y se llevó a cabo el procedimiento, sin complicaciones a corto ni largo plazo. Ambos contaron con remisión de la ML tanto clínica como en la ecografía Doppler, con cese de su sintomatología inicial. Conclusiones: Aún falta mucho conocimiento en el campo de las ML. El diagnóstico es clínico-imagenológico, y las ML del cayado del ducto torácico en particular suelen presentarse como una masa cervical baja. Estas debieran estudiarse idealmente como primer examen con ecografía Doppler y luego con resonancia magnética. Su manejo debe ser multidisciplinario, utilizando como primer paso la escleroterapia guiada por ultrasonido.
\end{abstract}

Palabras clave: Malformaciones linfáticas. Linfocele. Escleroterapia. Ducto torácico. Doxiciclina.

\section{Abstract}

Introduction: Macrocystic lymphatic malformations (LM) are low-flow vascular lesions characterized by disorganized lymphatic tissue and the formation of cystic cavities. Despite being benign, these can cause symptoms or complications, therefore requiring treatment. The initial management of choice is percutaneous sclerotherapy with ultrasound guidance. There are rare cases in which these lesions occur at the arch of the thoracic duct, presenting as a low cervical cystic mass. Objective: To describe, regarding two cases, the identification and management of LM located at the arch of the thoracic duct. Material and methods: A retrospective non-concurrent cohort analysis was performed, analyzing two cases of patients treated in our center with sclerotherapy with doxycycline and polidocanol for left supraclavicular LM. The diagnosis of these

Correspondencia:

*María S. Loyola-Zunino

E-mail: sloyola@med.puc.cl
Disponible en internet: 03-12-2021

Rev Chil Radiol. 2021;27(4):170-176 www. resochradi.com 0717-9308 / @ 2021 Sociedad Chilena de Radiología. Publicado por Permanyer. Éste es un artículo open access bajo la licencia CC BY-NC-ND (https://creativecommons.org/licenses/by-nc-nd/4.0/). 
and the technique used in the procedure are described in detail. Results: In both cases, the diagnosis of lymphatic malformation was confirmed, and the procedure was carried out without complications in the short or long term. Both had complete remission both clinically and on Doppler ultrasound, with ceasing of their initial symptoms. Conclusions: There is still a lack of knowledge concerning the topic of LM. Diagnosis is clinical-imaging, and LM of the arch of the thoracic duct, usually present themselves as a low cervical mass. These should ideally be studied initially with Doppler ultrasound and then with magnetic resonance imaging. The management of these should be multidisciplinary, using ultrasound-guided sclerotherapy as a first step.

Key words: Lymphatic malformations. Lymphocele. Sclerotherapy. Thoracic duct. Doxycycline.

\section{Introducción}

Las malformaciones vasculares de bajo flujo linfáticas quísticas (ML) son lesiones congénitas caracterizadas por tejido linfático desorganizado, que lleva a la dilatación de los conductos con acumulación de linfa y formación de cavidades quísticas, y pueden afectar a cualquier área anatómica'. La localización de las ML se distribuye, por frecuencia descendente, en la cabeza y el cuello $(48-75 \%)$, las extremidades $(20-42 \%)$ y otras áreas como el tronco o las vísceras $(10 \%)^{1-3}$. Pueden ser diagnosticadas al nacer 0 en forma tardía a cualquier edad. También existen lesiones adquiridas del sistema linfático, mayormente traumáticas, que pueden presentarse de forma similar ${ }^{4}$, o generarse de forma espontánea, y no ser posible identificar una causa ${ }^{5}$. Habitualmente se dividen en macroquísticas (más del $50 \%$ de los quistes tienen un tamaño $>2 \mathrm{~cm}$ ), microquísticas $(<2 \mathrm{~cm})$ y mixtas (menos del $50 \%$ de los quistes tienen un tamaño $>2 \mathrm{~cm})^{6,7}$.

Aunque son de naturaleza benigna, las ML pueden causar sintomatología por efecto de masa, con consecuencias funcionales y estéticas, siendo la de mayor gravedad la compresión de la vía aérea1. También pueden presentar otras complicaciones, entre las cuales las más frecuentes son las hemorragias y las infecciones ${ }^{8}$. Debido a esto, las ML suelen requerir tratamiento ${ }^{8}$.

El ducto torácico es la mayor estructura linfática del cuerpo, encargada de colectar la linfa y restituirla a la circulación venosa. Se origina en la cisterna del quilo, asciende retroaórtico en el tórax, forma un arco cervical y desemboca en la circulación venosa, con mayor frecuencia en la región subclavia izquierda, en la que también confluyen vasos linfáticos yugulares, subclavios y mediastínicos (Fig. 1). Las variantes anatómicas son muy habituales ${ }^{9}$. Con mayor frecuencia, la desembocadura se encuentra en la vena yugular interna izquierda 0 en el ángulo yugulosubclavio izquierdo. Otro sitio común es la vena subclavia izquierda e incluso puede haber desembocaduras múltiples

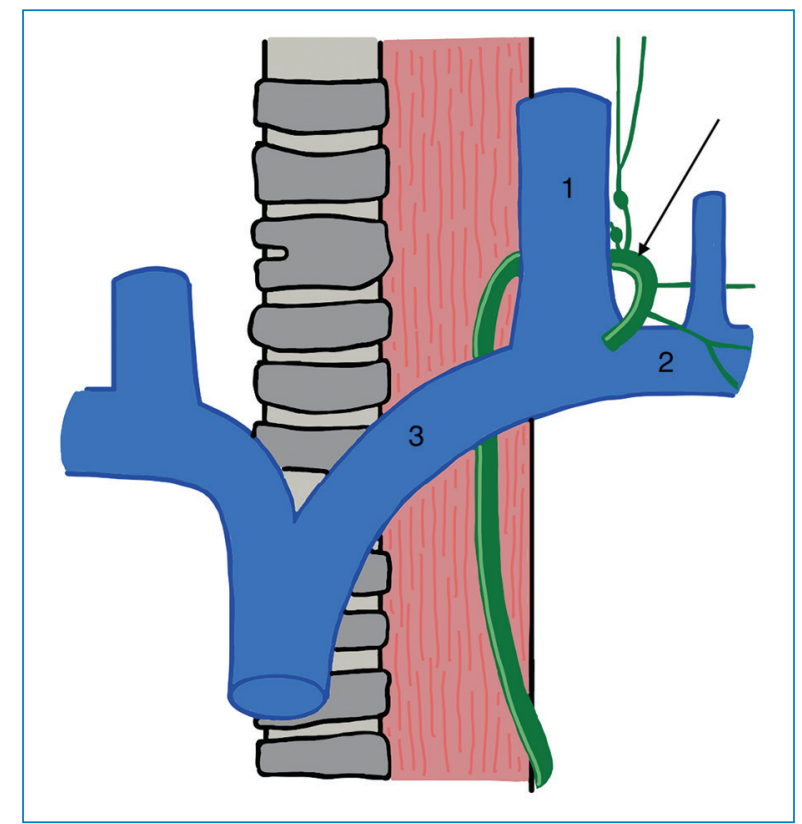

Figura 1. Trayecto final y desembocadura del ducto torácico (flecha) en la región del ángulo yugulosubclavio izquierdo. 1: vena yugular interna izquierda; 2: vena subclavia izquierda; 3 : vena braquiocefálica izquierda.

bilaterales ${ }^{9}$. El flujo de la linfa hacia la circulación está regulado por una válvula linfovenosa, la cual es de morfología, tamaño y ubicación variables ${ }^{10}$. Esta permite el paso de la linfa a la vena durante la inspiración y evita el reflujo de sangre durante la espiración, que aumenta la presión venosa central ${ }^{11}$.

Las dilataciones del cayado del ducto torácico son poco frecuentes ${ }^{12}$. Deben sospecharse en pacientes con aumento de volumen cervical bajo, especialmente izquierdo, que puede alcanzar un diámetro mayor de $10 \mathrm{~cm}$. Generalmente evolucionan con crecimiento lento, sin dolor ni complicaciones infecciosas ni hemorrágicas. En ocasiones se presentan con sintomatología secundaria a la compresión de estructuras adyacentes, como disnea, palpitaciones, edema local, disfagia u otras ${ }^{13}$. 
El manejo de las $\mathrm{ML}$ es multidisciplinario. El tratamiento depende de la sintomatología y de su naturaleza microquística o macroquística. La primera opción terapéutica en las ML macroquísticas es la escleroterapia, ya que es efectiva y poco invasiva, y presenta una baja tasa de complicaciones ${ }^{1,14}$.

La escleroterapia se realiza mediante una punción percutánea bajo guía ecográfica, que puede asociarse a fluoroscopía, para drenar el contenido de la ML e inyectar el esclerosante ${ }^{1,14}$. Este actúa dañando el endotelio de la ML, lo que causa posteriormente cicatrización y retracción de la lesión ${ }^{14}$. Existen diversos tipos de esclerosantes, pero entre los más utilizados en las ML se encuentran la doxiciclina, la bleomicina, el alcohol absoluto y sus derivados, como el polidocanol, el sotradecol y otros ${ }^{15}$. Pueden usarse de forma aislada o combinados según la disponibilidad de cada centro, las características y la ubicación de la lesión, los factores del paciente y la preferencia del operador.

El objetivo de este reporte es, a propósito de dos casos, describir el tratamiento mediante escleroterapia guiada por ecografía y fluoroscopía, con doxiciclina y polidocanol, en el manejo de ML macroquísticas ubicadas en relación con la desembocadura del ducto torácico. Esta ubicación es poco frecuente y existen pocos casos reportados en la literatura, tanto en el ámbito nacional como en el internacional, la mayoría tratados con cirugía. En este contexto, resulta de interés describir la identificación y el tratamiento mínimamente invasivo utilizado en nuestro centro de anomalías vasculares.

\section{Material y métodos}

Se realizó un análisis retrospectivo de cohorte no concurrente, accediendo a los registros electrónicos institucionales de los pacientes con diagnóstico de ML supraclavicular izquierda que fueron tratados con escleroterapia en nuestro centro hasta el año 2021, obteniendo dos pacientes con lesiones linfáticas quilosas. Ambos firmaron el consentimiento para difundir en forma anónima sus casos con fines científicos. Este proyecto fue revisado y aprobado por el Comité de Ética Institucional.

Frente al aumento de volumen cervical, un paciente fue evaluado mediante tomografía computada (TC) torácica y cervical con medio de contraste (Fig. 2), y el otro mediante resonancia magnética (RM) (Fig. 3). Los dos fueron diagnosticados como lesiones quísticas compatibles con ML.

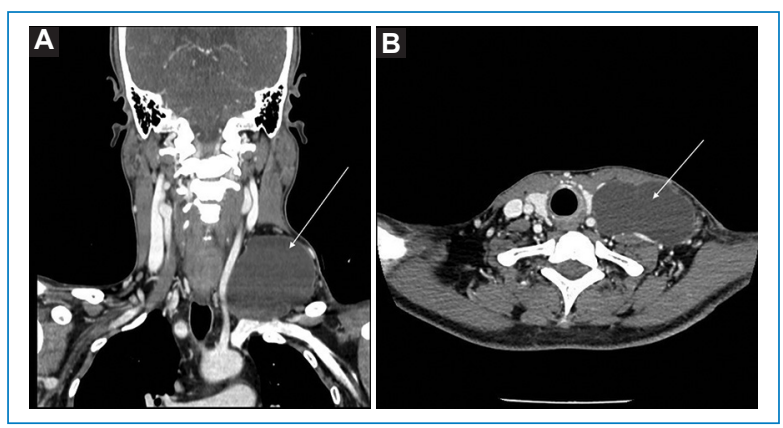

Figura 2. Tomografía computada de cabeza y cuello con contraste intravenoso, A: en cortes coronal y B: axial. Se observa la malformación linfática supraclavicular izquierda lobulada (flecha) con realce parietal de uno de los pacientes, previamente a la escleroterapia.

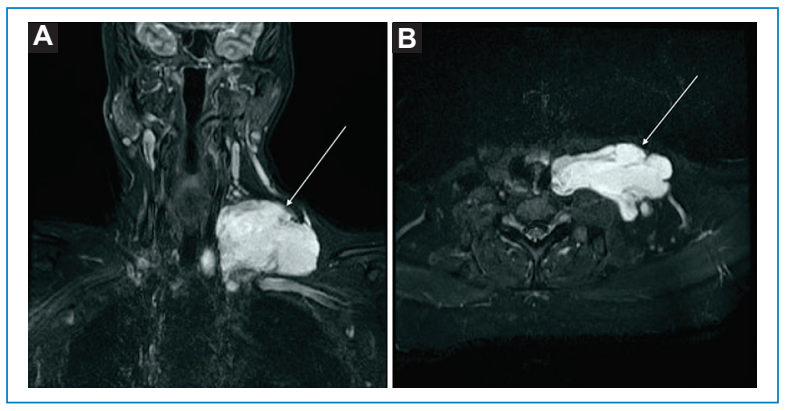

Figura 3. Resonancia magnética contrastada cervicotorácica en secuencias STIR, A: en cortes corona y B: axial. Se observa la malformación linfática supraclavicular izquierda (flecha) del segundo paciente, previamente a la escleroterapia, que aparece como una lesión polilobulada e infiltrante.

El tratamiento se realizó en el pabellón de terapia endovascular, bajo guía ecográfica y fluoroscópica, con medidas de antisepsia y bajo anestesia general. Se realizó una punción percutánea de la lesión quística con aguja de $24 \mathrm{G}$ bajo guía ecográfica. Se aspiró el contenido, que resultó lechoso, por lo cual se sospechó que correspondiera a quilo, y se envió una muestra al laboratorio. A continuación, se inyectó contraste yodado bajo fluoroscopía para obtener una representación de la cavidad de la lesión que debía ser tratada (Fig. 4).

En ambos casos las lesiones eran lobuladas, con tabiques parciales que permitieron contrastar completamente la cavidad. No hubo escape de contraste a la vena subclavia izquierda, por lo cual se consideró seguro efectuar la inyección de esclerosante. Se inyectó una mezcla 1:1 de $4 \mathrm{ml}$ de polidocanol líquido al $2 \%$ y medio de contraste, que se mantuvo por 


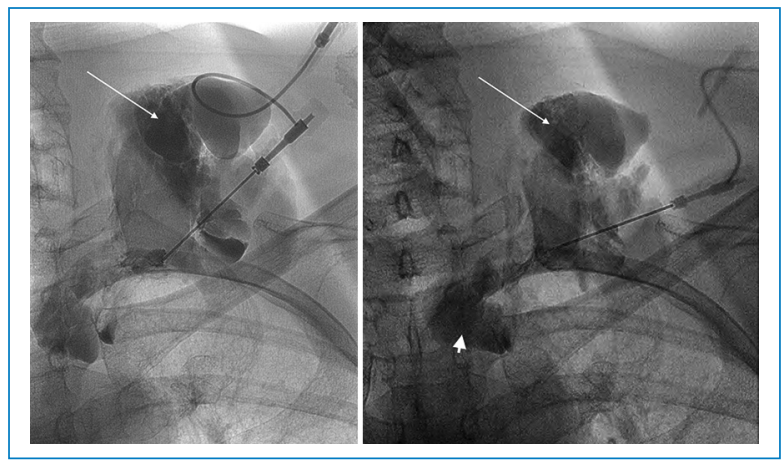

Figura 4. Fluoroscopía durante el procedimiento. Se observa el agente esclerosante mezclado con contraste yodado hidrosoluble inyectado dentro de la lesión (flecha), la cual presenta un componente intratorácico (punta de flecha). No se observó paso del contraste hacia las venas subclavia ni yugulares.

5 minutos, y luego se aspiró el contenido en forma parcial. Posteriormente se inyectó una ampolla de $100 \mathrm{mg}$ de doxiciclina en $5 \mathrm{ml}$, que se difundió dentro de la cavidad, usando el contraste residual de la cavidad como guía en la fluoroscopía. A continuación se retiró la aguja y se colocó un parche en la piel.

Los dos pacientes fueron controlados clínicamente a la semana y al mes postratamiento, y luego con ecografía Doppler de partes blandas a los 3 meses (Fig. 5).

\section{Resultados}

Se trataron dos pacientes, un hombre y una mujer de 46 y 54 años, respectivamente. Se realizaron el procedimiento y el seguimiento antes descritos.

Durante el procedimiento se extrajeron $210 \mathrm{ml}$ en un paciente y $110 \mathrm{ml}$ en el otro de líquido turbio amarillo compatible con quilo (Fig. 6), lo que se confirmó en el laboratorio por la alta concentración de triglicéridos en el aspirado. No se observaron filtraciones, lesiones de estructuras adyacentes ni otros incidentes en relación con el procedimiento. No hubo complicaciones secundarias a la escleroterapia, incluyendo efectos locales, complicaciones respiratorias, repercusión sistémica, necrosis de la piel, infección, cicatrices y cualquier otra morbilidad temporal o permanente relacionada con el procedimiento. Tampoco se registraron recurrencias de las lesiones tratadas ni mortalidad.

La evaluación de la respuesta a la escleroterapia se objetivó mediante su impacto en la sintomatología, la observación en la exploración física y la caracterización en las imágenes de ecografía Doppler. Ambos

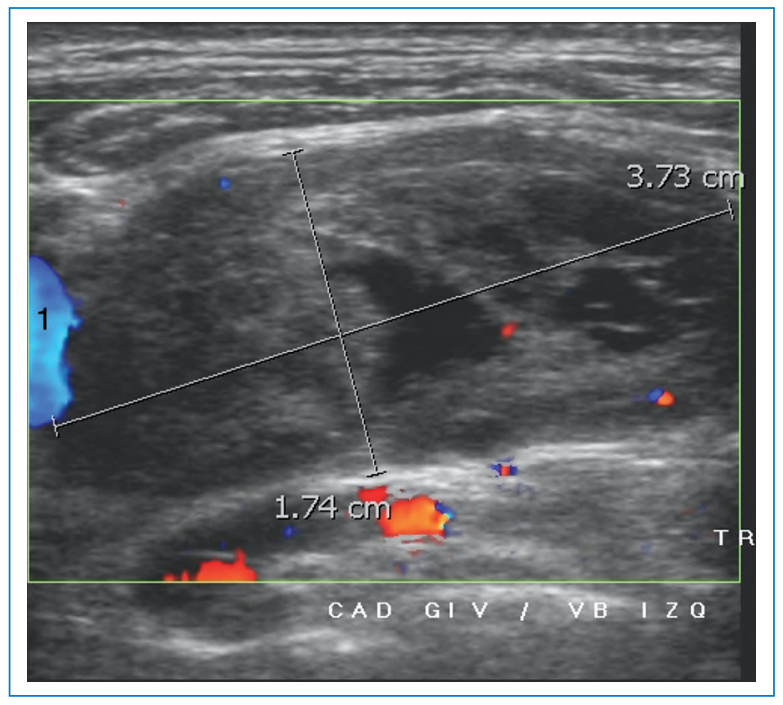

Figura 5. Ecografía Doppler de partes blandas en la región cervical izquierda, en plano transverso. Se observa, después del tratamiento, un nódulo sólido ecogénico (marcado entre los cursores de medición) adyacente y lateral a la vena yugular interna izquierda (1), de $3.7 \times 1.7 \mathrm{~cm}$ de diámetro, que corresponde al remanente cicatricial de la lesión esclerosada.

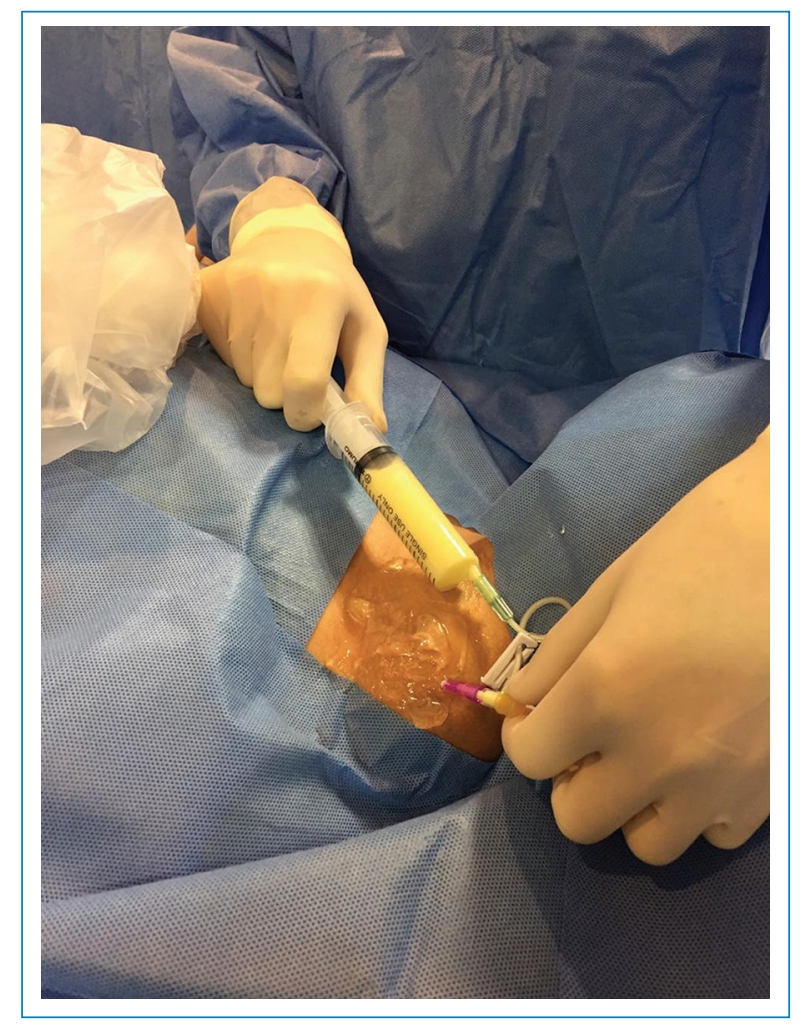

Figura 6. Líquido amarillo turbio compatible con quilo, aspirado de una de las lesiones quísticas supraclaviculares. 
pacientes presentaron remisión completa de la ML quilosa, con desaparición de la masa supraclavicular en la exploración clínica. Se utilizó como criterio imagenológico para definir una respuesta favorable la reducción de un $80 \%$ o más del tamaño original del quiste, criterio que ambos pacientes cumplieron en el control a los 3 meses. Dada la respuesta completa, en ambos casos se efectuó solo una sesión de escleroterapia.

\section{Discusión}

El diagnóstico diferencial de una masa quística supraclavicular izquierda incluye ML, linfocele adquirido, pseudoaneurismas, quistes de otra etiología y adenopatías con transformación quística.

Las ML son una patología poco frecuente que se presenta en 1 de cada 2000-4000 nacidos vivos ${ }^{16}$, aunque puede aparecer en forma más tardía a cualquier edad. Su conocimiento ha tenido un gran avance en los últimos años, tanto respecto a su etiología genética como a su manejo. Aunque el manejo es un campo en desarrollo, se han establecido algunos consensos. Uno de ellos es que el tratamiento debe ser multidisciplinario, ya que, dada su naturaleza benigna, no siempre es necesaria la extirpación como primer acercamiento terapéutico y el objetivo es el manejo sintomático que permita al paciente una buena calidad de vida. Otro consenso es que el tratamiento debe ser lo menos invasivo posible, inicialmente mediante escleroterapia, reservando la cirugía para aquellos pacientes en quienes la escleroterapia no es posible o no logra una buena respuesta. En este contexto, es de interés comunicar la respuesta favorable de este tipo de ML al tratamiento esclerosante, en especial considerando su ubicación en una zona anatómica compleja como es la desembocadura del ducto torácico.

Las ML cervicales se presentan como masas indoloras inespecíficas. Para su estudio, habitualmente la recomendación es realizar como examen inicial una ecografía de partes blandas, complementada con Doppler. La ecografía en modo B suele evidenciar una lesión anecogénica, con refuerzo acústico posterior, no compresible. Presentan algunos tabiques en su interior, en los cuales se puede observar al Doppler vascularización arterial o venosa en grado variable, siempre sin mecanismo de fístula. En casos de malformación linfática complicada con sobreinfección se puede ver un contenido de mayor ecogenicidad, asociado a cambios inflamatorios del tejido adyacente. En casos de hemorragia intralesional se observa contenido de mayor ecogenicidad, pudiendo formar niveles líquido-líquido con contenido ecogénico en la porción dependiente, correspondiente al contenido hemorrágico. Luego, para completar la caracterización de la lesión, su tamaño, vascularización y especialmente su extensión y su relación con estructuras profundas, se prefiere la RM contrastada o, en su defecto, la TC contrastada. Cuando existe sospecha de componente venoso o arterial en la lesión, la prueba de preferencia es la angiografía por RM. En general, las ML presentan bordes infiltrantes y septos, que se impregnan en forma tardía ${ }^{17}$. En los casos descritos, los pacientes fueron estudiados mediante TC (Fig. 2) o RM (Fig. 3), ya que no se sospechó inicialmente el diagnóstico de ML. La linfangio-RM dinámica es una técnica descrita en clínica en $2015^{18}$, en la que se realiza un estudio dinámico posterior a la inyección de gadolinio en los ganglios linfáticos inguinales. Es útil para la visualización de los linfáticos centrales, como la cisterna del quilo y el ducto torácico, especialmente en un contexto de extravasación de quilo en estos segmentos ${ }^{19}$. En nuestros pacientes podría haber sido de utilidad de haberse contrastado, demostrando así su relación con el ducto torácico, pero no se disponía de esta técnica en el momento del tratamiento de los pacientes reportados.

Cabe recalcar que la nomenclatura de consenso vigente (International Society for the Study of Vascular Anomalies) es «malformaciones vasculares de bajo flujo linfáticas» y no «linfangiomas». Esto se debe a que la palabra «linfangioma» puede inducir al error de considerarlas tumores linfáticos, lo cual es incorrecto, ya que el índice mitótico no se encuentra aumentado?.

$\mathrm{Al}$ evaluar ML supraclaviculares, especialmente las izquierdas, es importante considerar dentro del diagnóstico diferencial que pueden estar originadas o en relación con la desembocadura del ducto torácico. La linfangio-RM puede ser de utilidad para establecer este origen. La presentación tardía en ambos casos es menos frecuente (aunque las ML pueden hacerse clínicamente evidentes a cualquier edad). Esto podría haber hecho sospechar que fueron lesiones adquiridas («linfoceles»); sin embargo, no se estableció una lesión causal en estos casos. Otro diagnóstico que es necesario descartar es que correspondan a patologías proliferativas.

Con respecto al tratamiento, aunque la literatura reporta pocos casos, la mayoría de los descritos fueron manejados quirúrgicamente ${ }^{5,12,13,20-22}$. En nuestro centro, sin embargo, se trató como primera opción con escleroterapia, que fue efectiva en estos dos pacientes con ML con conexión al ducto torácico. 
Para el tratamiento de las $\mathrm{ML}$, asociadas o no al ducto torácico, existen distintos agentes esclerosantes y para su elección hay que considerar múltiples factores, que incluyen, entre otros, el tipo de malformación, la edad del paciente, la efectividad, los efectos adversos, la disponibilidad de los agentes, y la experiencia y la preferencia de los operadores. En nuestros pacientes se utilizaron doxiciclina y polidocanol, buscando un efecto sinérgico al combinar sus mecanismos de acción.

La doxiciclina es un antibiótico que intralesionalmente ha demostrado tener efecto esclerosante en las $\mathrm{ML}$, además de contar con un buen perfil de seguridad, con baja incidencia de efectos adversos. Al ser un derivado de la tetraciclina, se ha descrito tinción dental en niños menores de 8 años sin dentición definitiva $a^{1,6,14}$. $\mathrm{Su}$ mecanismo esclerosante exacto se desconoce, pero se cree que está asociado a la inhibición del crecimiento y el desarrollo enzimático local, además de ser proinflamatorio ${ }^{15}$. El polidocanol, al ser un derivado del alcohol, posee un efecto detergente, que causa daño y posterior retracción y fibrosis endotelial. Esto permite eliminar la linfa y el quilo para aumentar la exposición del endotelio a la acción del esclerosante. De esta forma, el polidocanol podría actuar como coadyuvante del efecto de la doxiciclina en el tratamiento de estas lesiones.

En ambos casos descritos, el tratamiento fue efectivo en las ML del ducto torácico cervical. En general, es necesario realizar escleroterapia seriada para manejar las ML, ya que con frecuencia los tabiques no permiten la comunicación de todas las cavidades que conforman la malformación. La escleroterapia suele requerir, en promedio, tres o cuatro sesiones. En estos dos casos fue suficiente una sesión para lograr la regresión clínica de las lesiones. Esto podría explicarse por la acción combinada de los agentes esclerosantes y el tipo de lesión macroquística, con tabiques parciales que permitieron tratar la lesión completa. Las ML macroquísticas tienen en general mejores tasas de curación que las microquísticas ${ }^{1,6,23}$, debido a la mayor y más fácil exposición del endotelio a los fármacos y al menor componente de tejido sólido, lo que permite una mayor disminución del volumen de la lesión.

A modo de conclusión, se requiere mayor conocimiento con respecto a las $\mathrm{ML}$ quilosas y su manejo. Nuestro estudio está limitado por la cantidad de pacientes, ya que dos casos no permiten establecer significancia estadística ni recomendaciones clínicas. Sin embargo, pretende aportar evidencia respeto a la efectividad de la escleroterapia guiada con ecografía y fluoroscopía. Para establecer el diagnóstico, primero debe existir una sospecha clínica. Luego las ML requieren ser estudiadas y caracterizadas adecuadamente con métodos de imagen ampliamente disponibles. El estudio inicial se realiza mediante ecografía Doppler y luego con RM o TC se busca una caracterización más detallada y la relación con las estructuras anatómicas adyacentes para, según lo observado, planificar la estrategia de tratamiento. La linfangio-RM puede ser de utilidad para confirmar la comunicación de las ML con el ducto torácico. Existen alternativas de tratamiento percutáneo efectivas y con una importante mejoría en la calidad de vida de los pacientes, como es la escleroterapia. Se discuten dos casos de ML quilosas en relación con la desembocadura del ducto torácico, que son poco frecuentes en la práctica médica, y resulta interesante describir su presentación, estudio por imágenes, manejo percutáneo mediante escleroterapia y favorable respuesta terapéutica obtenida con esta técnica.

\section{Financiamiento}

No se recibió financiamiento externo.

\section{Conflicto de intereses}

Los autores declaran no tener conflictos de intereses.

\section{Responsabilidades éticas}

Protección de personas y animales. Los autores declaran que los procedimientos seguidos se conformaron a las normas éticas del comité de experimentación humana responsable y de acuerdo con la Asociación Médica Mundial y la Declaración de Helsinki.

Confidencialidad de los datos. Los autores declaran que han seguido los protocolos de su centro de trabajo sobre la publicación de datos de pacientes.

Derecho a la privacidad y consentimiento informado. Los autores han obtenido el consentimiento informado de los pacientes y/o sujetos referidos en el artículo. Este documento obra en poder del autor de correspondencia.

\section{Bibliografía} 1. Acord M, Srinivasan AS, Cahill AM. Percutaneous treatment of lymphatic
malformations. Tech Vasc Interv Radiol. 2016; 19 (4): 305-311
2. Alqahtani A, Nguyen LT, Flageole H, Shaw K, Laberge JM. 25 years' ex-
perience with lymphangiomas in children. J Pediatr Surg. 1999;34:1164-8. 
Rev Chil Radiol. 2021;27(4)

3. Shergill A, John P, Amaral JG. Doxycycline sclerotherapy in children with lymphatic malformations: outcomes, complications and clinical efficacy. Pediatr Radiol. 2012;42:1080-8.

4. Chantarasak DN, Green MF. Delayed lymphocoele following neck dissection. Br J Plast Surg. 1989;42:339-40.

5. Ray J, Braithwaite D, Patel PJ. Spontaneous thoracic duct cyst. Eur Arch Otorhinolaryngol. 2003;260:280-2.

6. De Maria L, De Sanctis P, Balakrishnan K, Tollefson M, Brinjikji W. Sclerotherapy for lymphatic malformations of head and neck: systematic review and meta-analysis. J Vasc Surg Venous Lymphat Disord. 2020;8:154-64.

7. ISSVA Classification for Vascular Anomalies. (Acceso el 05-04-2020). Disponible en: https://www.issva.org/UserFiles/file/ISSVA-Classification-2018.pdf.

8. Perkins JA, Manning SC, Tempero RM, Cunningham MJ, Edmonds JL, Hoffer FA, et al. Lymphatic malformations: review of current treatment. Otolaryngol Head Neck Surg. 2010;142:795-803.

9. Phang KL, Bowman M, Phillips A, Windsor J. Review of thoracic duct anatomical variations and clinical implications. Clin Anat. 2014;27:637-44.

10. O'Hagan LA, Windsor JA, Phillips ARJ, Itkin M, Russell PS, Mirjalili SA Anatomy of the lymphovenous valve of the thoracic duct in humans. J Anat. 2020;236:1146-53.

11. Ratnayake CBB, Escott ABJ, Phillips ARJ, Windsor JA. The anatomy and physiology of the terminal thoracic duct and ostial valve in health and disease: potential implications for intervention. J Anat. 2018;233:1-14.

12. Lecanu JB, Gallas D, Biacabe B, Bonfils P. Lymphocele of the thoracic duct presenting as a left supraclavicular mass: a case report and review of the literature. Auris Nasus Larynx. 2001;28:275-7.
13. Abelardo $E$, Shastri $P$, Prabhu V. Variations in the management of cervical thoracic duct cyst. Biomed Hub. 2020;5:7-14.

14. Gurgacz S, Zamora L, Scott NA. Percutaneous sclerotherapy for vascular malformations: a systematic review. Ann Vasc Surg. 2014;28:1335-49.

15. Horbach SER, Lokhorst MM, Saeed P, De Goüyon Matignon De Pontouraude CMF, Rothová A, Van Der Horst CMAM. Sclerotherapy for low-flow vascular malformations of the head and neck: a systematic review of sclerosing agents. J Plast Reconstr Aesthet Surg. 2016:69:295-304.

16. Kennedy TL, Whitaker M, Pellitteri P, Wood WE. Cystic hygroma/lymphangioma. A rational approach to management. Laryngoscope. 2001;111:1929-37.

17. Hamilton BE, Nesbit GM, Gross N, Andersen P, Sauer D, Harnsberger HR. Characteristic imaging findings in lymphoceles of the head and neck. Am J Roentgenol. 2011;197:1431-5.

18. Krishnamurthy R, Hernandez A, Kavuk S, Annam A, Pimpalwar S. Imaging the central conducting lymphatics: initial experience with dynamic MR lymphangiography. Radiology. 2015;274:871-8.

19. Dori Y. Novel lymphatic imaging techniques. Tech Vasc Interv Radiol. 2016;19:255-61.

20. Hekiert A, Newman J, Sargent R, Weinstein G. Spontaneous cervical lymphocele. Head Neck. 2007;29:77-80.

21. Chapman KR, Ratnatunga PC. Thoracic duct cyst presenting as a left supraclavicular mass. Ceylon Med J. 2001;46:28

22. Gupta M, Lovelace TD, Sukumar M, Gosselin MV. Cervical thoracic duct cyst. J Thorac Imaging. 2005;20:107-9.

23. Wiegand S, Eivazi B, Zimmermann AP, Sesterhenn AM, Werner JA Sclerotherapy of lymphangiomas of the head and neck. Head Neck. 2011;33:1649-55. 